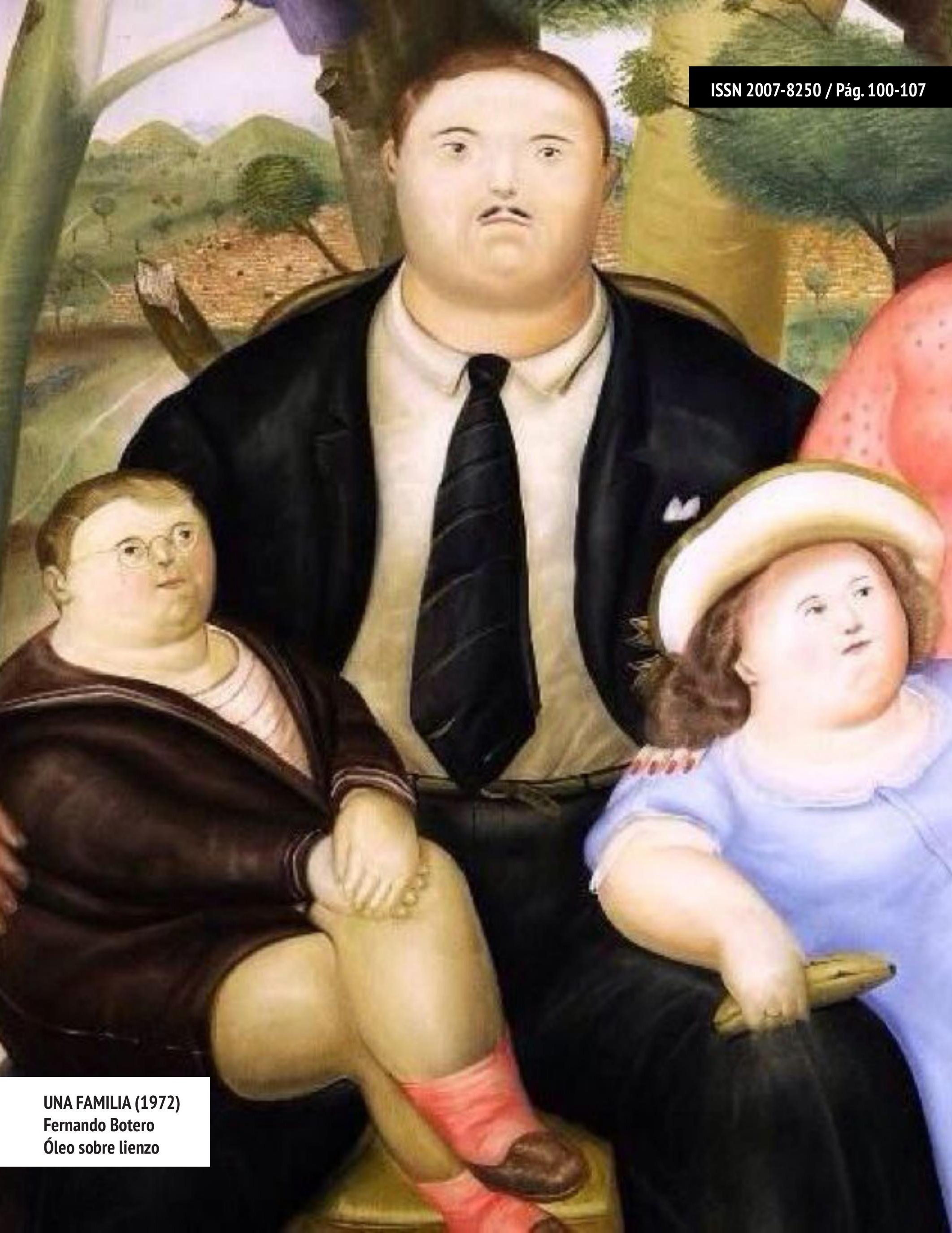




\section{PROGRAMA DE INCLUSIÓN EDUCATIVA Y SOCIAL}

\section{EDUCATIONAL AND SOCIAL INCLUSION PROGRAM}

\section{RESUMEN}

El Programa de Inclusión Educativa y Social comienza a gestarse en el año 2016 y opera a partir de agosto de 2018 en la Preparatoria 8 de la Universidad Autónoma de Nuevo León. Nace como un proyecto para brindar a los alumnos con barreras para el aprendizaje y la participación una estadía satisfactoria en el nivel medio superior y evitar la reprobación escolar apostando a la inclusión. Es un programa que se fundamenta en el trabajo colaborativo y transversal; alineado a referentes institucionales y nacionales; $y$ respaldado por redes de apoyo y personal capacitado. El Programa de Inclusión Educativa y Social tiene relación con todas las Unidades de Aprendizaje del bachillerato; cada docente recibe un reporte de recomendación de cada uno de los alumnos identificados, se cuenta con el apoyo de los practicantes de la ENEHRL (Escuela Normal de Especialización Humberto Ramos Lozano), mediante entrevistas y evaluaciones psicopedagógicas se identifican necesidades de cada alumno mismas que son comunicadas al maestro para la elaboración de estrategias de aprendizaje adaptadas al alumno. Este programa de Inclusión Educativa y Social tiene un impacto positivo en todas las unidades de aprendizaje; el docente recibe una constante capacitación para identificar las necesidades de los alumnos que participan en el programa y de esta manera adapta estrategias de aprendizaje para ayudar al desempeño de los alumnos que presentan barreras para el aprendizaje y la participación.

PALABRAS CLAVE: Inclusión - Educación - Diversidad - Equidad - Aprendizaje - Programal.

\section{CLAUDIA ROMERO GAONA GABRIELA ALICIA PIÑA GONZÁLEZ}

The Educational and Social Inclusion Program begins to take shape in 2016 and started in August 2018. It was born as a project to provide students with barriers to learning and participation a satisfactory stay at the High school level and avoid school failure betting on inclusion. It is a program that is based on collaborative and transversal work; aligned with institutional and national references; and backed by support networks and trained staff. The Educational and Social Inclusion Program is related to all the High School Learning Units; Each teacher receives a recommendation report from each of the identified students, they have the support of the practitioners of the ENEHRL (Humberto Ramos Lozano Specialization Normal School), through interviews and psychopedagogical evaluations, the needs of each are identified and communicated to the teacher for the development of learning strategies adapted to the student. This program of Educational and Social Inclusion has a positive impact on all learning units; The teacher receives constant training to identify the needs of the students who participate in the program and in this way adapts learning strategies to help the performance of students who present barriers to learning and participation.

KEYWORDS: Inclusion - Education - Diversity - Equity - Learning - Program. 
L

a Preparatoria 8 de la Universidad Autónoma de Nuevo León a través de la coordinación del departamento de Tutorías y con el apoyo del departamento de Orientación creó el Programa de Inclusión Educativa y Social con la finalidad de brindar oportunidad educativa a alumnos identificados con barreras para el aprendizaje y la participación.

Aunque el Programa de Inclusión Educativa y Social se puso en marcha en agosto de 2018, se gestó desde el 2016; fecha a partir de la cual, se inició un proceso de sensibilización y concientización con todo el personal de la Preparatoria 8 con el objetivo de reforzar los conceptos de diversidad e inclusión.

Se entiende por diversidad a la diferencia 0 a la distinción entre personas, animales o cosas, a la variedad, a la infinidad 0 a la abundancia de cosas diferentes, a la desemejanza, a la disparidad 0 a la multiplicidad. De acuerdo con la UNESCO (2017), la inclusión es un enfoque que responde positivamente a la diversidad de las personas y a las diferencias individuales, entendiendo que la diversidad no es un problema, sino una oportunidad para el enriquecimiento de la sociedad, a través de la activa participación en la vida familiar, en la educación, en el trabajo y en general en todos los procesos sociales, culturales y en las comunidades.

Un programa de inclusión contribuye a mejorar la cobertura, inclusión y equidad educativa entre toda la población (Rodríguez, 2019). El Programa de Inclusión Educativa y Social de la Preparatoria 8 tiene las siguientes características:
- Se fundamenta en el trabajo colaborativo y transversal.

- Alineado a referentes institucionales y nacionales.

- Respaldado por redes de apoyo y personal capacitado.

Además se busca fortalecer las siguientes competencias genéricas con la totalidad de alumnos que tiene la dependencia a través de estrategias de concientización y sensibilización:

1. Se conoce y valora a sí mismo y aborda problemas y retos teniendo en cuenta los objetivos que persigue.

2. Mantiene una actitud respetuosa hacia la interculturalidad y la diversidad de creencias, valores, ideas y prácticas sociales.

\section{ATRIBUTOS}

1. Enfrenta las dificultades que se le presentan y es consciente de sus valores, fortalezas y debilidades.

- Identifica sus emociones, las maneja de manera constructiva y reconoce la necesidad de solicitar apoyo ante una situación que lo rebase.

- Elige alternativas y cursos de acción con base en criterios susten- tados y en el marco de un proyecto de vida.

- Analiza críticamente los factores que influyen en su toma de decisiones.

- Asume las consecuencias de sus comportamientos y decisiones.

- Administra los recursos disponibles teniendo en cuenta las restricciones para el logro de sus metas.

2. Reconoce que la diversidad tiene lugar en un espacio democrático de igualdad de dignidad y derechos de todas las personas, y rechaza toda forma de discriminación.

- Dialoga y aprende de personas con distintos puntos de vista y tradiciones culturales mediante la ubicación de sus propias circunstancias en un contexto más amplio.

- Asume que el respeto de las diferencias es el principio de integración y convivencia en los contextos local, nacional e internacional.

\section{DISCIPLINARES}

- H13. Analiza y resuelve de manera reflexiva problemas éticos relacionados con el ejercicio de

\section{De acuerdo con la UNESCO (2017), la inclusión} es un enfoque que responde positivamente a la diversidad de las personas y a las diferencias individuales, entendiendo que la diversidad no es un problema, sino una oportunidad para el enriquecimiento de la sociedad 
su autonomía, libertad y responsabilidad en su vida cotidiana.

- H14. Valora los fundamentos en los que se sustenta los derechos humanos y los practica de manera crítica en la vida cotidiana.

- H15. Sustenta juicios a través de valores éticos en los distintos ámbitos de la vida.

- H1. Analiza y evalúa la importancia de la filosofía en su formación personal y colectiva.

- H3. Examina y argumenta de manera crítica y reflexiva diversos problemas filosóficos relacionados con la actuación humana, potenciando su dignidad, libertad y autodirección.

Con los alumnos que participan en el Programa de Inclusión Educativa y Social se busca que fortalezcan las siguientes competencias genéricas:

1. Se conoce y valora a sí mismo y aborda problemas y retos teniendo en cuenta los objetivos que persigue.

2. Elige y practica estilos de vida saludables.

3. Aprende por iniciativa e interés propio a lo largo de la vida.

4. Participa y colabora de manera efectiva en equipos diversos.

\section{ESTRATEGIA DIDÁCTICA}

El Programa de Inclusión es un plan de trabajo sistemático que atiende a alumnos que presentan barreras para el aprendizaje y la participación, el cual busca la inclusión de los mismos en el Nivel Medio Superior (Preparatoria 8) con el objetivo de evitar la deserción 0 el rezago escolar. Se le da al alumno un seguimiento personalizado, a través de nuestras redes de apoyo (Fapsi, Inclusión UANL, CUS, ENEHRL).

\section{AMBIENTE DE APRENDIZAJE}

Se genera un ambiente de aprendizaje favorable y armónico para los alumnos que presentan barreras para el aprendizaje y la participación. El programa de inclusión trabaja sobre los contenidos conceptuales, procedimentales y actitudinales de cada Unidad de Aprendizaje, es decir, los contenidos establecidos en el Programa Analítico no se alteran.

El docente de cada Unidad de Aprendizaje realiza un trabajo colaborativo con los practicantes de la ENEHRL y realizan la adaptación de las estrategias de enseñanza-aprendizaje, sin modificar los contenidos de la malla curricular. Las diferencias individuales no son un problema, sino una oportunidad para el enriquecimiento de la sociedad, a través de la participación activa en la vida familiar, en la educación, en el trabajo y en general en todos los procesos sociales, culturales y en las comunidades (Thomas, G. 2002).

La educación inclusiva tiene que ver con remover todas las barreras para el aprendizaje, y facilitar la participación de todos los estudiantes vulnerables a la exclusión y la marginalización. Significa que todos los estudiantes reciben los soportes que requieren para tener la oportunidad de participar como miembros de una clase 0 aula regular, con pares de su misma edad y de contribuir a sus colegios del vecindario.

\section{MÉTODO}

1. Sensibilización y capacitación a docentes, personal administrativo y de apoyo reforzando los conceptos de diversidad e inclusión.

2. Seguimiento personalizado de los alumnos a través de:
- Entrevistas y juntas con los Padres de Familia.

- Taller de Inclusión.

- Consejería psicológica.

- Apoyo pedagógico a alumnos.

La metodología del programa abarca desde el pre ingreso del alumno, canalizándolo a la Coordinación de Inclusión Educativa para personas con Discapacidad y Adultos Mayores de la UANL, quienes registran a los alumnos para brindar el día del examen de asignación de espacios un apoyo personalizado.

Se realiza una primera entrevista con los padres de familia de los alumnos que hayan alcanzado el puntaje en el examen de asignación de espacios e ingresarán con nosotros a la preparatoria; el objetivo de dicha entrevista es que los padres de familia nos brinden información sobre recomendaciones específicas para trabajar con el alumno, estas recomendaciones específicas se entregan a cada uno de los maestros que les darán clase. El departamento de Orientación se encarga de entregar a los orientadores infogramas de los diversos diagnósticos y da seguimiento a los alumnos en la parte académica para dar canalización al programa de fortalecimiento académico y apoyo escolar para que reciban asesorías preventivas en las áreas que haya que reforzar.

El trabajo con los padres de familia inicia con un curso taller donde se les informa de todos los servicios con los que cuenta el alumno y se les brindan charlas y citas continuas para dar seguimiento al rendimiento académico del alumno. El seguimiento personalizado de los alumnos identificados con barreras para el aprendizaje consiste 
Tabla 1. Datos sobre la cantidad de alumnos inscritos al programa y que lograron avanzar al siguiente semestre

Total de alumnos inscritos en el 18 programa

Total de alumnos que lograron avanzar al I4 siguiente semestre

Total de alumnos que no lograron 4 avanzar al siguiente semestre

\section{Programa de Inclusión Educativa y Social} Semestre: Agosto - Diciembre 2018

- Total de alumos que lograron avanzar al siguiente semestre

- Total de alumnos que no lograron avanzar al siguiente semestre
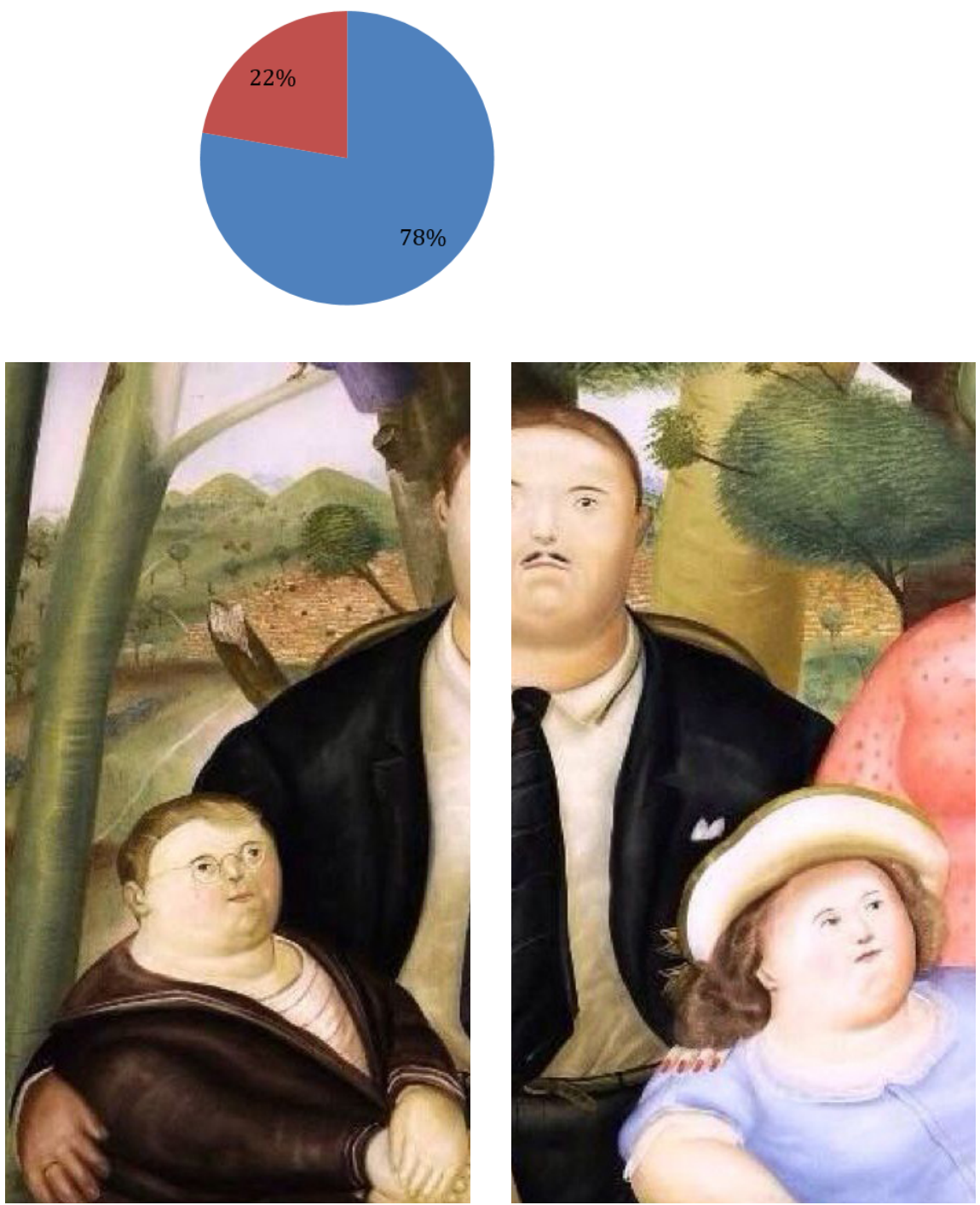

Presencia Universitaria

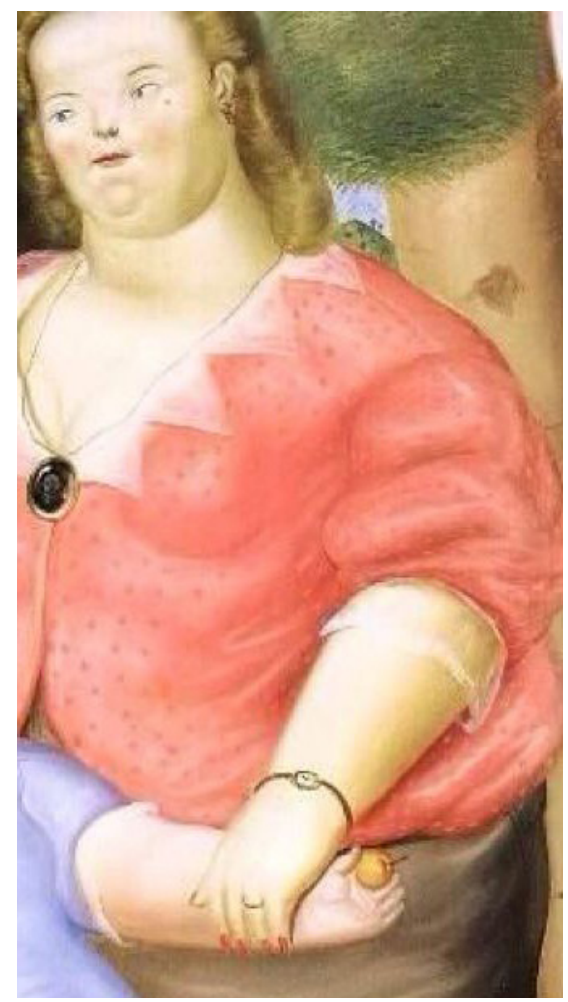

Tabla 2. Muestra el total de alumnos lograron avanzar al siguiente semestre

Total de alumnos inscritos en el 14 programa

Total de alumnos que lograron avanzar al $\quad 14$

siguiente semestre

Total de alumnos que no lograron 0

avanzar al siguiente semestre

\section{Programa de Inclusión Educativa y Social Semestre: Enero - Junio 2019}

- Total de alumos que lograron avanzar al siguiente semestre

- Total de alumnos que no lograron avanzar al siguiente semestre

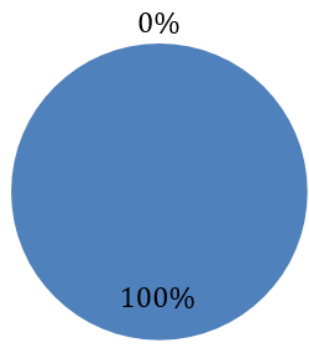

Preparatoria 8 UANL 
en talleres para el desarrollo de habilidades socioemocionales y motoras, sensibilizar y concientizar a su grupo sobre la condición de vida que tiene, medir su desempeño al final de semestre (avance de semestre, integración, socialización, etc).

Durante la estancia en la Preparatoria 8 se cuenta con el apoyo de psicólogos de la FaPsi (Facultad de Psicología de la UANL) para la consejería de los alumnos y con el apoyo de practicantes de la Escuela Normal de Especialización Humberto Ramos Lozano para el apoyo académico del alumno.

\section{RECOMENDACIONES}

Al implementar un programa de Inclusión se debe considerar primeramente la sensibilización y concientización de todo el personal que labora en la Dependencia, en especial de los Docentes quienes trabajan frente a frente con los alumnos, además de definir y capacitar a las personas que estarán dirigiendo el Programa.

Además de las recomendaciones antes mencionadas, también es de mucha ayuda la búsqueda de instituciones y/o escuelas, para hacer un trabajo colaborativo y se unan esfuerzos con el mismo objetivo. También es importante darle seguimiento al Programa y de alguna manera evaluar los resultados que se obtienen, con el fin de hacer adecuaciones necesarias en las áreas de oportunidad que se vayan detectando.

\section{RESULTADOS}

El impacto de la operación del Programa de Inclusión Educativa y Social en la Preparatoria 8 se puede observar desde el punto de vista cuantitativo y cualitativo. En cuanto al porcentaje de avance académico, en el semestre Agosto - Diciembre 2018 se contaba con un total de 18 alumnos detectados con barreras para el aprendizaje, de los cuales 14 lograron avanzar al siguiente semestre. A los 4 alumnos que de alguna manera quedaron rezagados se les siguió brindando ayuda y siguieron siendo monitoreados con el objetivo de reinsertarlos de nuevo a la educación. Se muestra en la Tabla 1 los resultados.

Al finalizar el Semestre Agosto - Diciembre 2018 se realizó una evaluación sobre los resultados obtenidos y se detectaron las fortalezas y áreas de oportunidad del programa obteniendo estrategias que se pusieron en marcha en el siguiente semestre inmediato. Se contaba con 14 alumnos identificados con barreras para el aprendizaje, los cuales el $100 \%$ lograron avanzar al siguiente semestre. En la Tala 2 se muestran los resultados obtenidos

\section{CONCLUSIÓN}

Con este programa la Preparatoria 8 de la Universidad Autónoma de Nuevo León abre paso de forma exitosa a la diversidad y a la educación. A través del Programa de Educación Inclusiva se cumplieron los objetivos de brindar a los alumnos con barreras para el aprendizaje y la participación una estadía satisfactoria en el nivel medio superior, evitando el rezago académico y la deserción escolar.

Su metodología fundamentada en el trabajo colaborativo y transversal está alineado a referentes institucionales y nacionales y respaldado por redes de apoyo y personal capacitado. En el presente artículo presentamos la metodología que se llevó a cabo, los resultados y las redes de apoyo con las que actualmente seguimos trabajando. Destacamos que el Programa de Inclusión de la Preparatoria 8 fue el primer programa en presentarse ante la DFIE Dirección de Formación Integral al Estudiante, el cual ha sido reconocido como un referente institucional que promueve la diversidad y la inclusión.

Creemos que lo que hasta ahora se ha logrado es perfectible y estamos conscientes de que la inclusión es un proceso que traerá beneficios en nuestra dependencia en cuanto a el respeto, la comprensión y la atención a la diversidad cultural, social e individual, el acceso en condiciones de igualdad a una educación de calidad, buscando que la escuela sea un lugar de encuentro donde se derriben las barreras que propician la discriminación y se celebre la diversidad, además de que promueva el acceso, la permanencia, la atención, la participación y el logro de todos (Rodríguez, 2019).

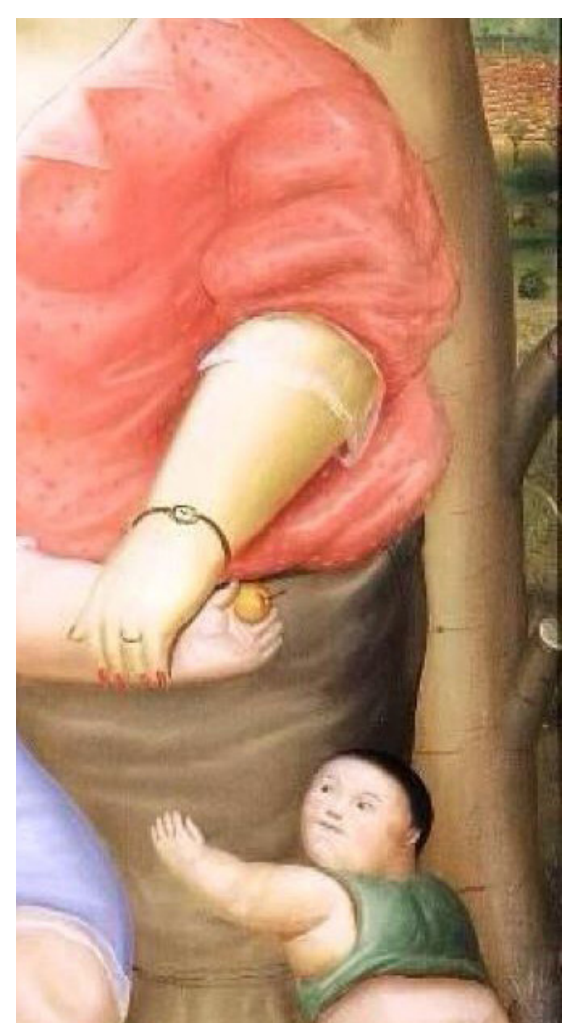




\section{REFERENCIAS}

Rodríguez, H (2019). La Educación Inclusiva en la Agenda Educativa Mundial 2015-2030.

UNESCO (2017). Guía para asegurar la inclusión y la equidad en la educación. Manual del Sistema Nacional de Educación Media Superior (SINEMS).

Modelo Educativo (Equidad e Inclusión) de la SEP, 1era. Edición, 2017 (sems. gob.mx).

Thomas, G. (2002). El Síndrome de Asperger: estrategias prácticas para el aula: guía para el profesorado 1.a ed. - Vitoria-Gasteiz: Servicio Central de Publicaciones del Gobierno Vasco.

Convención sobre los Derechos de las Personas con Discapacidad. Nueva York y Ginebra, 2008. Naciones Unidas.

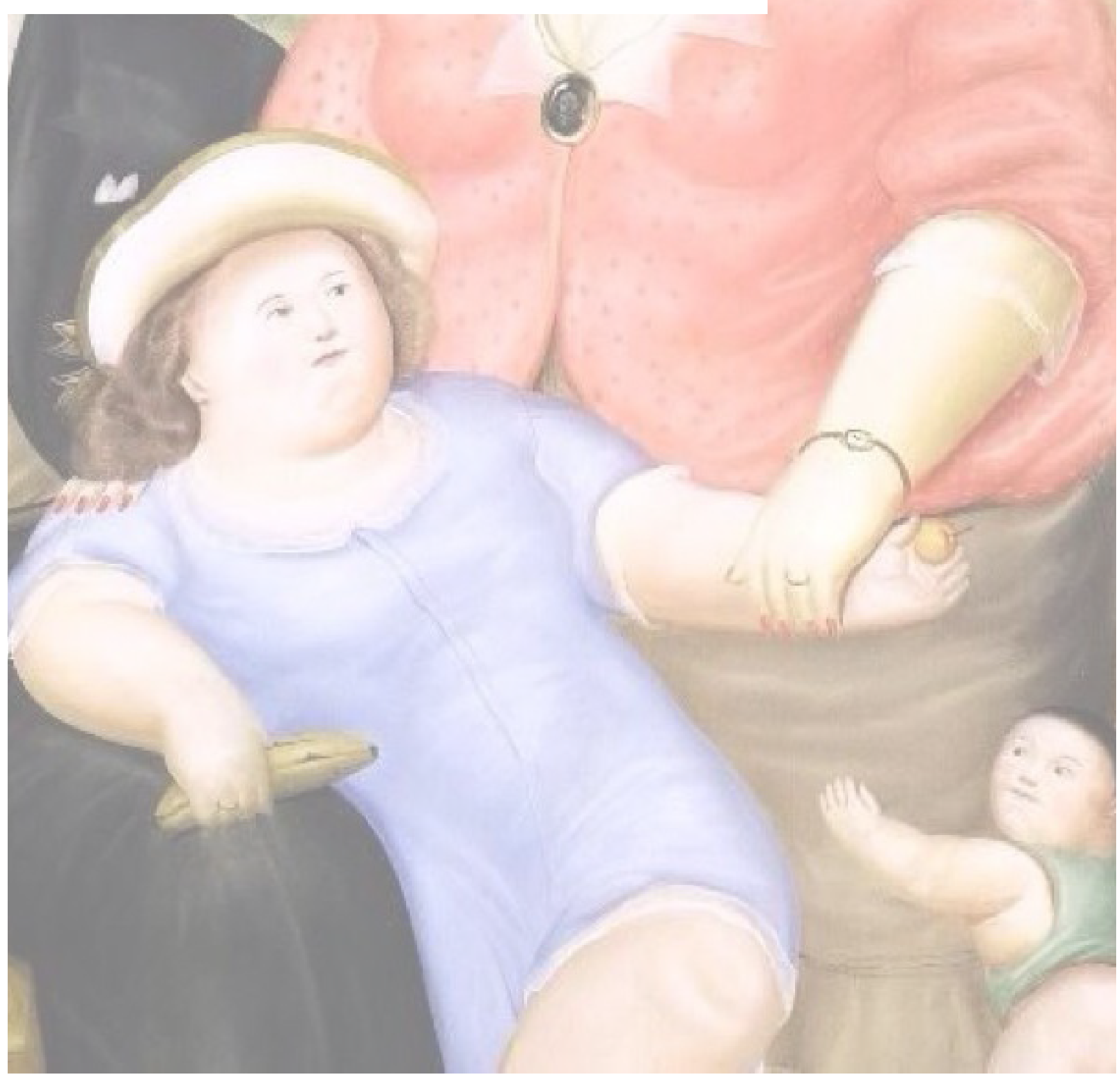




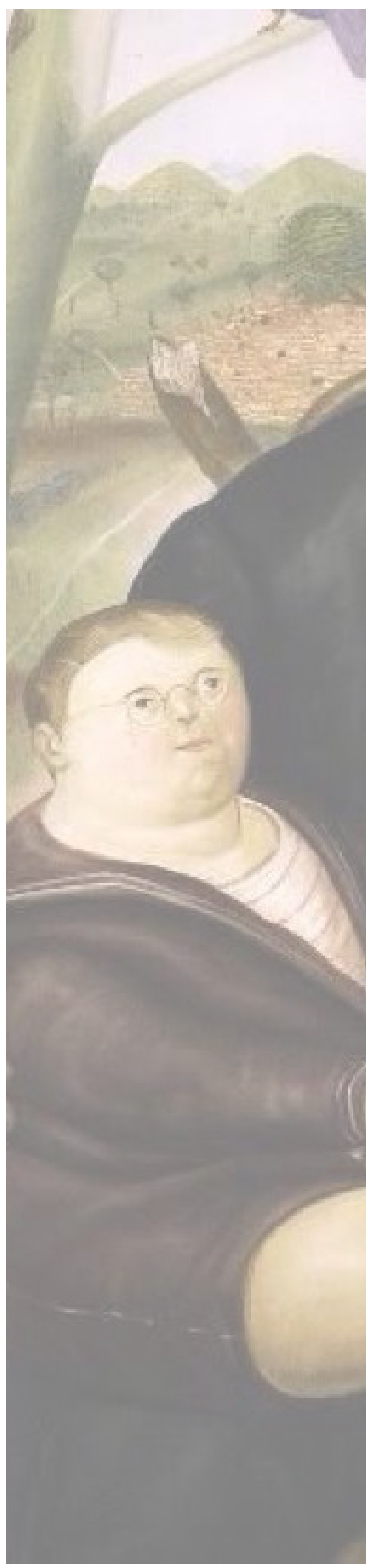

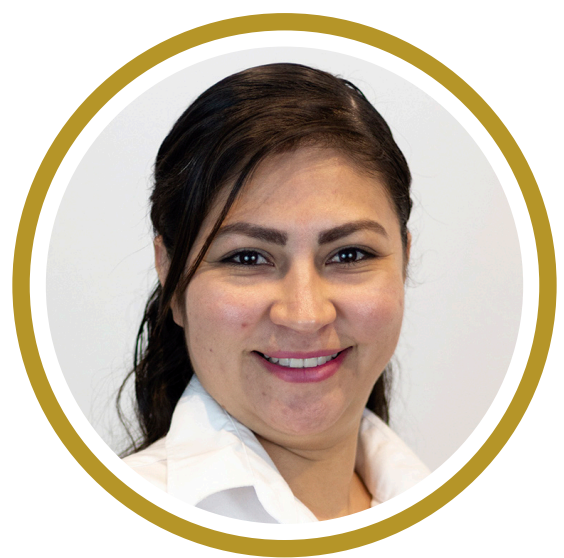

\section{Claudia Romero Gaona}

Es egresada de la Licenciatura en Psicología, actualmente cursa el nivel de Maestría en la Escuela Normal de Especialización Humberto Ramos Lozano. Al momento es Docente de las unidades de aprendizaje La Vida en México y Filosofía, y es coordinadora del Departamento de Tutorías de la Preparatoria 8 de la UANL.

\section{Correo Electrónico:}

clau_romero_05@hotmail.com

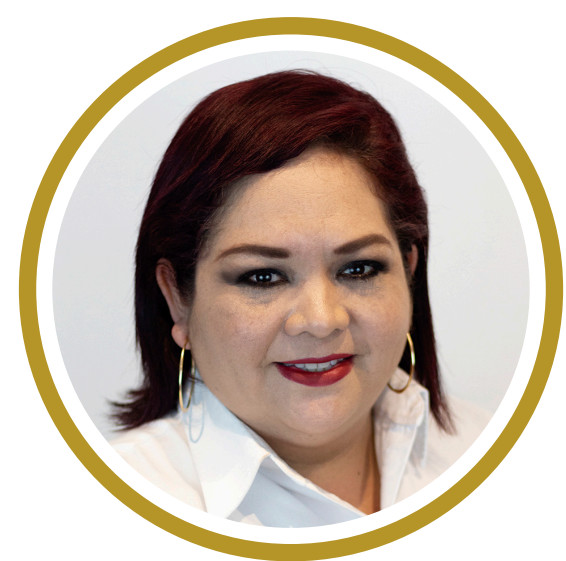

Gabriela Alicia Piña González

Es egresada de la Licenciatura de Educación, cuenta con una Maestría en Educación Basada en Competencias. Actualmente es Docente en las unidades de aprendizaje Expresión Oral y Escrita y Compresión, y Expresión Lingüística Avanzada, y es Jefa de Personal del Departamento de Orientación Psicopedagógica de la Preparatoria 8 de la UANL.

\section{Correo Electrónico:}

gapinag@hotmail.com

Fecha de Recepción: 15/03/20

Fecha de Aprobación: 15/08/20 